\title{
Anticancer Effects of Baicalein in FRO Thyroid Cancer Cells Through the Up-regulation of ERK/p38 MAPK and Akt Pathway
}

\author{
SE EUN HAN*, CHAN HO PARK*, IL SUNG NAM-GOONG, YOUNG IL KIM and EUN SOOK KIM \\ Department of Internal Medicine, Ulsan University Hospital, \\ University of Ulsan College of Medicine, Ulsan, Republic of Korea
}

\begin{abstract}
Background/Aim: The aim of the study was to evaluate the anticancer effects of baicalein in FRO anaplastic thyroid cancer (ATC) cells. Materials and Methods: FRO cells were treated with baicalein and viability was measured by the MTT assay. Cell apoptosis was observed by staining with Hoechst dye. The expression of apoptotic proteins (Bax, Bcl-2, PARP, cytochrome c, and caspase-3) and the inflammatory protein Cox-2 and the phosphorylation of MAPKs and Akt were determined by western blot. Results: Treatment with baicalein inhibited cell proliferation in a time-dependent manner and increased DNA fragmentation and apoptosis in FRO cells. Baicalein at 50 and $100 \mu M$ inhibited the expression of Bax, PARP, cytochrome $c$, cleaved caspase-3, and Cox-2, and increased the expression of Bcl-2. Baicalein increased the phosphorylation of ERK, p38 MAPK, and Akt and decreased JNK phosphorylation. Conclusion: Baicalein caused anticancer effects in FRO ATC cells through induction of apoptosis and regulation of the MAPK and Akt pathway.
\end{abstract}

Thyroid is closely related to the central nervous system function, especially the fight or flight response and plays a role in the homeostasis of the body. Thyroid hormone controls the metabolism respiration and heart function. Therefore, increased levels of thyroid hormone result in troubles in breathing, swallowing, and sleep, heart

This article is freely accessible online.

*These Authors contributed equally to this study.

Correspondence to: Eun Sook Kim, MD, Ph.D., Department of Internal Medicine, Ulsan University Hospital, Ulsan 682-714, Republic of Korea. Tel: +82 522508837, Fax: +82 522507048, e-mail: hanseeun0521@gmail.com and endo10@daum.net

Key Words: Baicalein, anaplastic thyroid cancer, FRO cell, apoptosis, MAPK/Akt pathway. malfunction, nervousness, warmness, hunger and weight loss. Low levels of thyroid hormone induce chronic fatigue and weight gaining. Imbalance of thyroid hormones is related with thyroid disorders such as hyperthyroidism, hypothyroidism, and thyroid cancer.

Thyroid cancer develops when thyroid cells grow uncontrollably, but the cause of the transformation of thyroid cells to cancer cells is not known yet (1). Thyroid cancer cells can grow in to two different tumors, benign and malignant. Benign thyroid cancer enlarges the thyroid gland, changes its shape and produces lumps and bumps which are called thyroid nodules. These nodules overproduce thyroid hormone and cause hyperthyroidism. Malignant thyroid tumors can be divided into three categories: differentiated thyroid cancers, which develop from thyroid follicular cells, medullary thyroid carcinoma, which accounts for $4 \%$ of thyroid cancers but can spread to lymph nodes, lungs or liver, and the anaplastic thyroid cancer, which is also called anaplastic carcinoma and accounts for $2 \%$ of thyroid cancers. The anaplastic thyroid cancer is also called undifferentiated and often spreads into the neck and other parts of the body quickly (2).

To treat thyroid cancer, different methods should be chosen according to the type and the stage of cancer: surgery, radioactive iodine treatment, thyroid hormone therapy, external-beam radiation therapy, chemotherapy, and targeted therapy. Two or more of these options may be combined to enhance therapeutic capacity $(3,4)$. However, chemotherapy could cause various side-effects depending on the type and dose of drugs and may also lead to cardiovascular disease through accumulation of cardiac toxicity (5). Therefore, to avoid side-effects, natural products from many different sources such as plants, marine organisms and microorganisms are considered for the treatment of cancer (6).

Baicalein (5, 6, 7-trihydroxyflavone) is a bioactive flavone, originally isolated from the roots of Scutellaria baicalensis and Scutellaria lateriflora which are traditionally used to treat various inflammatory diseases. In particular, it 


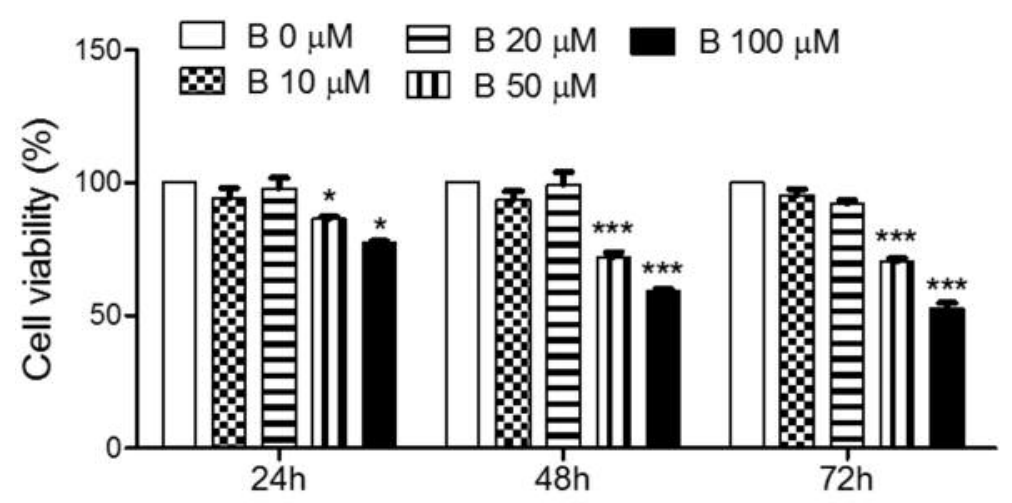

Figure 1. Effects of baicalein on the viability of FRO cells. Cells were treated with 10, 20, 50, and $100 \mu \mathrm{M}$ baicalein for 24, 48, and $72 \mathrm{~h}$. The cell viability was determined by MTT assay. The data are presented as mean $\pm S . E . M$ of three independent experiments. $* p<0.05$ and $* * * p<0.001$ vs. untreated cells.

has been shown that baicalein the displays anticancer properties against various cancers such as pancreatic cancer $(7,8)$, ovarian cancer (9), and gastric cancer (10) through the induction of cancer cell apoptosis, cell-cycle arrest, inhibition of angiogenesis, metastasis, and inflammation (11, 12). Thus, baicalein has great potential as an agent for the treatment and prevention of cancer without causing severe side effects. In the present study, to evaluate the therapeutic potential of baicalein in ATC, its anticancer effects were investigated in FRO cells.

\section{Materials and Methods}

Chemicals. Baicalein, dimethyl sulfoxide, and anti- $\beta$-Actin monoclonal antibody (mAb) were purchased from Sigma Aldrich (St Louis, MO, USA). The anti- Akt, phospho-Akt cleavedcaspase-3, cytochrome $c$, ERK, p-ERK, JNK, p-JNK, p38, p-p38 MAPK, PI3K mAbs were purchased from Cell signaling (Boston, MA, USA). The anti- Bcl-2 and procaspase-3 mAbs were purchased from Santa Cruz Biotechnology (Santa Cruz, CA, USA). The anti-COX-2 mAb was purchased from Cayman Chemicals (Ann Arbor, MI, USA). The anti-PARP mAb was from Anaspec Inc (Fremont, CA, USA). The HRP-conjugate goat antirabbit-IgG and goat anti-mouse-IgG were from Biorad (Hercules, CA, USA). RIPA buffer was from Thermo Scientific Co. (Rockford, IL, USA), 1X protease inhibitor cocktail tissue 2 perfect was from Quartett (Berlin, Germany), and Xpert phosphatase inhibitor was from Gendepot (Barker, TX, USA). The nitrocellulose (NC) membrane and Clarity western ECL substrate were purchased from Bio-Rad (Hercules, CA, USA). X-ray film was from AGFA Co. (Mortsel, Belgium).

Cell culture. FRO cells, a human anaplastic thyroid carcinoma cells, were cultured in RPMI-1640 medium (Corning, Manassas, VA, USA) supplemented with $10 \%$ fetal bovine serum (FBS; Dublin, CA, USA) and $1 \%$ penicillin/streptomycin solution $(10,000 \mathrm{U} / \mathrm{ml}$, Thermo Fisher Scientific, MA, USA). The cells were maintained in in a humid atmosphere at $37^{\circ} \mathrm{C}$ with $5 \% \mathrm{CO}_{2}$.
Cell viability assay. Cell viability was determined by MTT Cell Proliferation Assay Kit I (Roche Diagnostics GmbH, Mannheim, Germany). FRO cells cultured in 96 -well plates $\left(5 \times 10^{4} /\right.$ well $)$ were treated with baicalein $(0,10,20,50$, and $100 \mu \mathrm{M})$ and then incubated in a humid atmosphere at $37^{\circ} \mathrm{C}$ with $5 \% \mathrm{CO}_{2}$ for 24,48 , and $72 \mathrm{~h}$. At the end of each time period, the medium was replaced with $5 \mathrm{mg} / \mathrm{well}$ of MTT solution and incubated for $4 \mathrm{~h}$. The reaction was stopped by removing the MTT solution and adding solvent solution into each well for $10 \mathrm{~min}$ at room temperature (RT) while shaking. The solubilized purple formazan crystals were transferred into 96 -well plate $(100 \mu \mathrm{l} /$ well $)$ and the color was measured by reading the optical density (O.D.) with a microplate reader (UVM, Cambridge, UK) at $570 \mathrm{~nm}$.

Western blotting. The cells were treated with baicalein $(0,20,50$, and $100 \mu \mathrm{M}$ ) for $48 \mathrm{~h}$, harvested and then lysed using RIPA Lysis and Extraction Buffer (Thermo Fisher Scientific) containing 1x protease inhibitor cocktail 2 perfect and Xpert phosphatase inhibitor (GenDEPOT, TX, USA). Cell debris were removed by centrifugation (Micro 171TR. Hanil scientific Inc, Kimpo, Republic of Korea) at $16,000 \times g$ for $20 \mathrm{~min}$ at $4^{\circ} \mathrm{C}$. The protein lysate was separated by electrophoresis on $10 \%$ SDS polyacrylamide gels (Biorad, Hercules, CA, USA). The proteins were transferred in $0.2 \mu \mathrm{m}$ nitrocellulose (NC) membranes (Biorad). Membranes were incubated in 5\% skim milk dissolved in $1 \times$ TBS-T $(10 \mathrm{mM}$ Tris base, $100 \mathrm{mM} \mathrm{NaCl}$, and $0.05 \%$ Tween20) for $3 \mathrm{~h}$ at RT for blocking, each membrane was then incubated with primary monoclonal antibodies (mAbs) overnight $(\mathrm{O} / \mathrm{N})$ at $4^{\circ} \mathrm{C}$. The membranes were washed with $1 \mathrm{x}$ TBS-T for $15 \mathrm{~min}$ for 5 times, and then source matched anti-rabbit or anti-mouse mAbs in $5 \%$ skim milk were added for $3 \mathrm{~h}$ at RT. The membranes were washed 5 times with $1 \mathrm{x}$ TBS-T for 15 min developed with ECL solution and analyzed using Image Lab Software for PC ver. 5.2.1. (Bio-Rad Image System). To evaluate the relative protein expression levels, the bands of Bcl-2, Bax, pro-caspase-3, cleavedcaspase- 3 , cytochrome $c$, PARP, and PI3K were compared to those of $\beta$-actin and ratio was calculated. The phosphorylated forms of ERK, p38, JNK, and Akt were calculated in relation to their total forms. The quantitation was performed by Image $\mathrm{J}$ program ver. 1.47 (NIH, USA). 


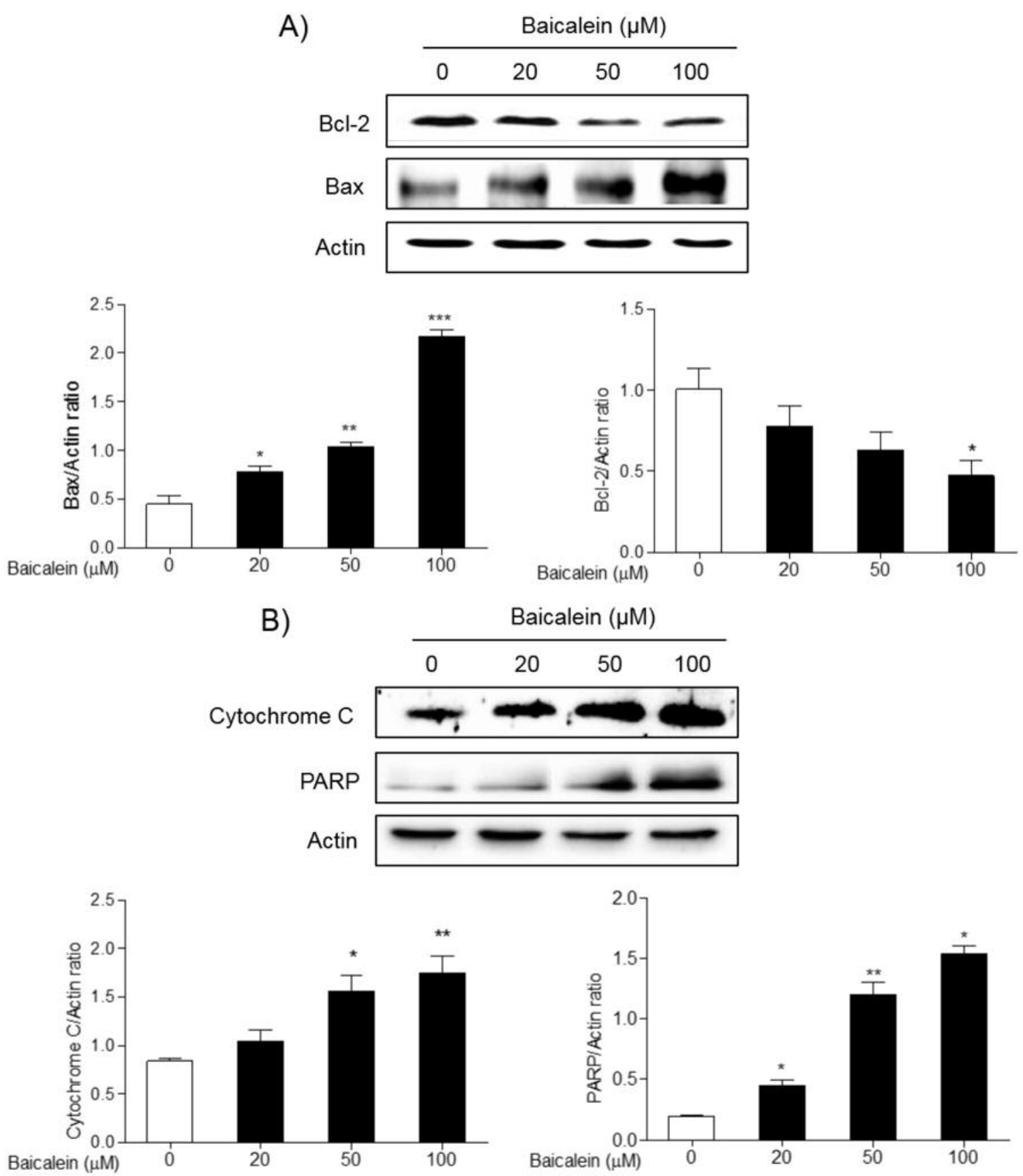

Figure 2. Effects of baicalein on the expression of Bcl-2, Bax, cytochrome c, and PARP proteins in FRO cells. Cells were treated with 20, 50, and $100 \mu \mathrm{M}$ baicalein for $48 \mathrm{~h}$, and the expression of Bcl-2, Bax (A), cytochrome c, and PARP (B) was determined by western blotting. Actin was used as internal loading control. Data are presented as mean \pm S.E.M of the ratio of the intensity of bands of the above proteins $v s$. the intensity actin bands of three independent experiments. ${ }^{*} p<0.05,{ }^{*} p<0.01$ and $* * * p<0.001$ vs. normal cells.

Hoechst staining. The cells were cultured in glass dishes (SPL Life Science, Gyeonggi-do, Korea) and treated with baicalein $(0,20,50$ and $100 \mu \mathrm{M}$ ) for $48 \mathrm{~h}$. Following treatment, cells were washed with $1 \times$ PBS three times, and incubated with the Hoechst 33342 Solution (Thermo Fisher Scientific for $15 \mathrm{~min}$. The stained cells were washed three times with 1x PBS and observed under fluorescent microscope (Leica, Wetzlar, Germany).

Statistical analysis. All experimental data were expressed as mean \pm standard deviation (SD) using Graphpad Prism 5.0 (GraphPad 

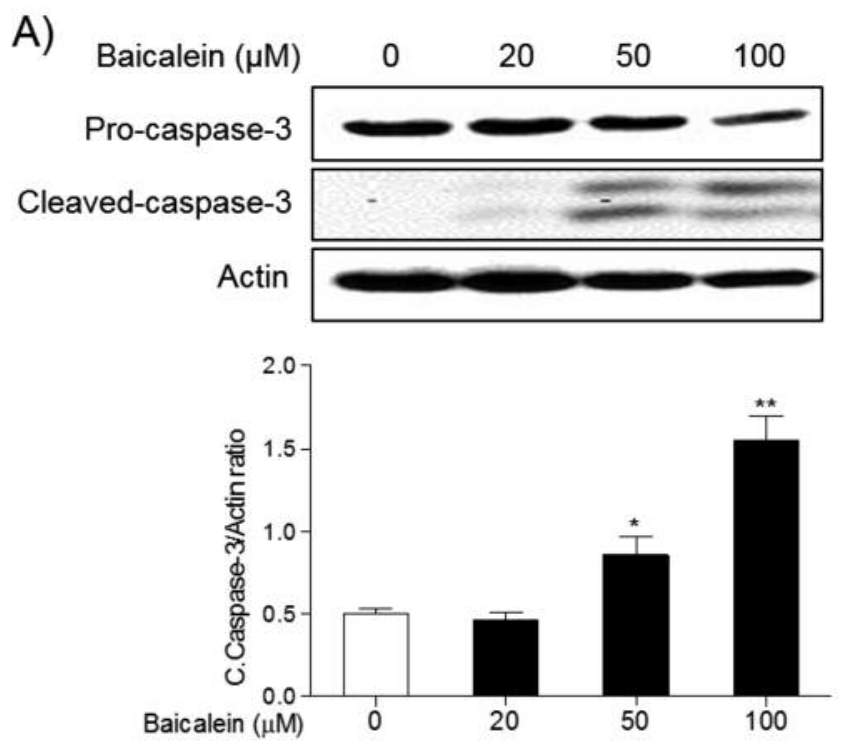

B)
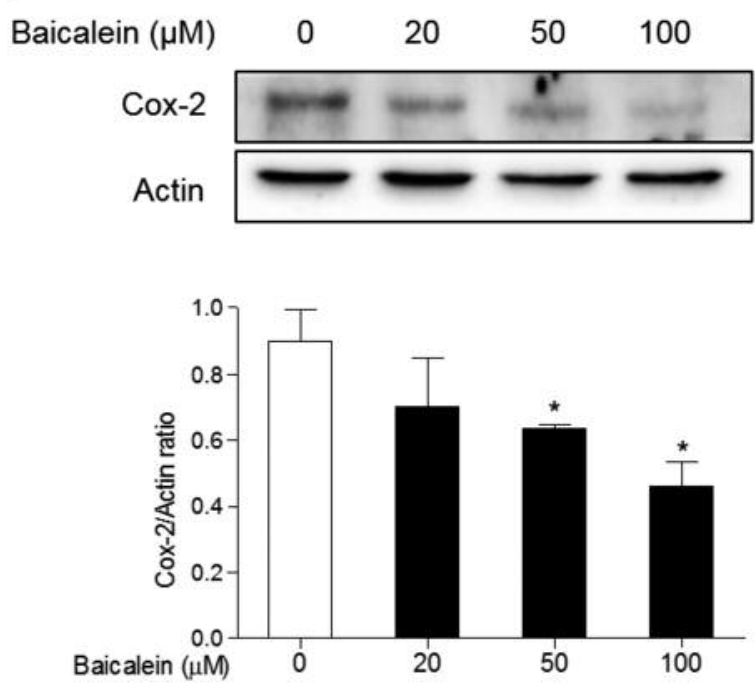

Figure 3. Effects of baicalein on the expression of caspase-3 and Cox-2 proteins in FRO cells. Cells were treated with 20 , 50 , and 100 MM baicalein for $48 \mathrm{~h}$, and the expression of caspase-3 (A), and Cox-2 (B) was determined by western blotting. Actin was used as internal loading control. Data are presented as mean \pm S.E.M of the ratio of the intensity of bands of the above proteins vs. the intensity of the actin bands of three independent experiments. *p<0.05, and **p<0.01 vs. normal cells.

Software, La Jolla, CA, USA). Statistical analysis was performed using GraphPad Prism program ver. 5.00 (GraphPad Software Inc., CA, USA). The group were compared using Student's t-test and one-way ANOVA and $p<0.05$ was considered statistically significant.

\section{Results}

The effects of baicalein on cell proliferation. To determine the effect of baicalein on cell proliferation, the cell viability was measured by MTT. As shown in Figure 1, treatment with baicalein significantly decreased the viability of FRO cells in a time and concentration dependent manner. The low doses of baicalein, 10 and $20 \mu \mathrm{M}$, did not affect cell viability; however, higher concentrations of baicalein, 50 and $100 \mu \mathrm{M}$, significantly reduced the cell proliferation.

The effects of baicalein on the expression of apoptotic proteins. To investigate the effects of baicalein on the apoptosis of cancer cells, the expression of apoptotic proteins in FRO cells was examined by western blot. Treatment 20, 50 , and $100 \mu \mathrm{M}$ baicalein significantly increased the expression of pro-apoptotic proteins, Bax, cytochrome $c$, and PARP, while decreased the expression of anti-apoptotic protein, Bcl-2, in a concentration-dependent manner (Figure 2). In addition, treatment 50 and $100 \mu \mathrm{M}$ baicalein significantly increased the expression of cleaved caspase-3 compared to normal cells, but significantly decreased the expression of inflammatory protein Cox-2 in FRO cells (Figure 3).
Next, anti-apoptotic effects of baicalein were investigated by the observation of morphological changes of Hoechst nuclear staining in FRO cells b. As shown in Figure 4, treatment with baicalein increased DNA fragmentation in a dose dependent manner.

The effects of baicalein on the apoptotic signaling pathway. To understand the mechanism of action of baicalein on the apoptosis of FRO cells, its effects on MAPK and Akt/PI3K signaling pathways were investigated by western blot. Treatment of FRO cells with 50 and $100 \mu \mathrm{M}$ baicalein significantly induced phosphorylation of ERK (Figure 5A) and p38 MAPK (Figure 5B), while reduced the phosphorylation of JNK MAPK (Figure 5C). Meanwhile, the phosphorylation of Akt was decreased in a concentration dependent manner $d$ (Figure 6A). Inhibition was is significant at $100 \mu \mathrm{M} / \mathrm{ml}$ baicalein. However, the expression of PI3K was not changed in FRO cells (Figure 6B). These results indicate that baicalein can induce apoptosis in FRO cells through activation of the ERK/p38 MAPK, and partially, PI3K signaling pathways.

\section{Discussion}

Thyroid cancer is the most common type of endocrine cancer. Although ATC corresponds only to $2 \%$ of thyroid cancers, it is a poorly differentiated and tends to invade the surrounding tissues such as trachea. ATC has an early onset and is resistant 

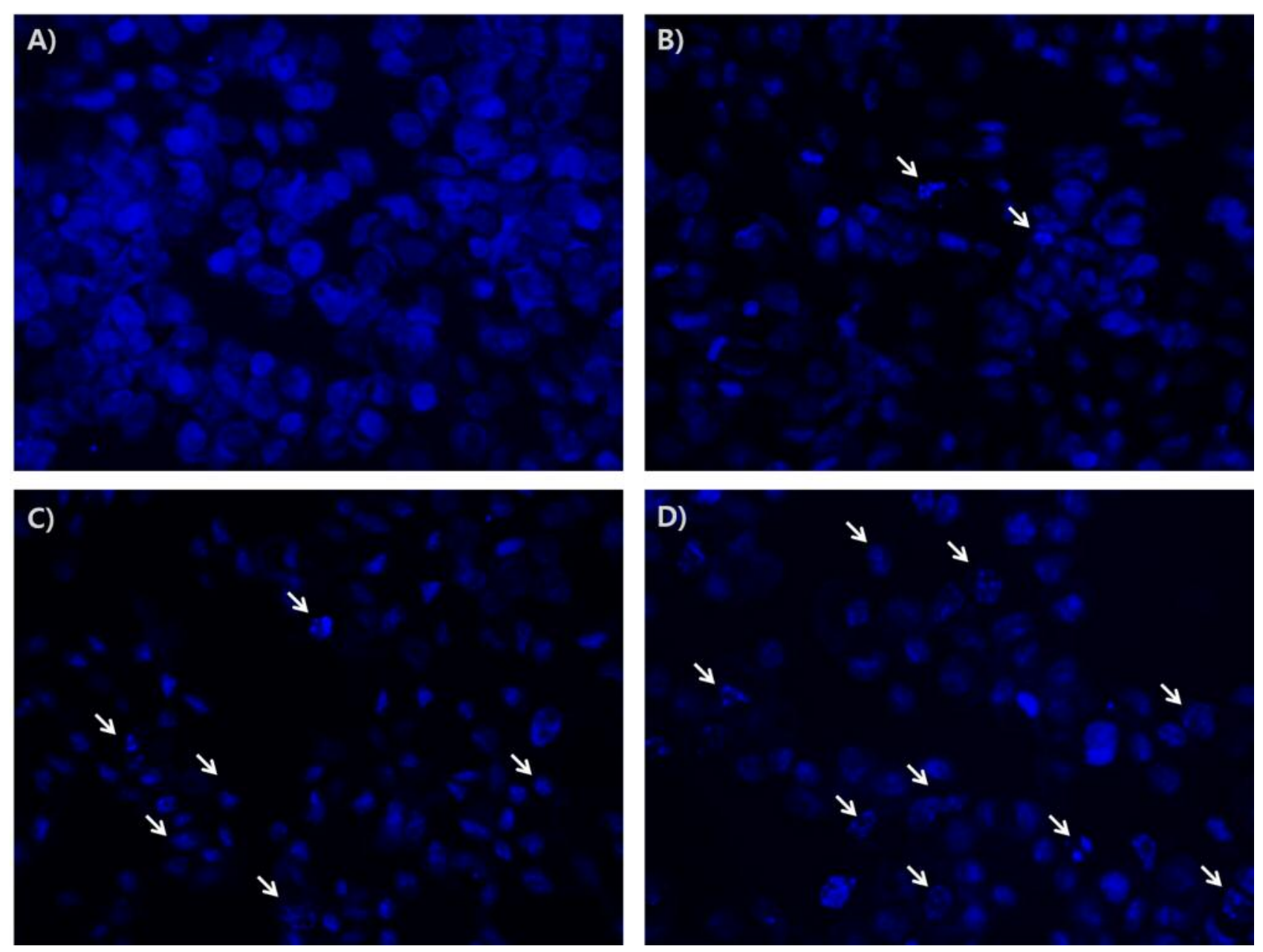

Figure 4. Effects of baicalein on the apoptosis in FRO cells. Cells were treated with 20, 50, and $100 \mu$ M baicalein for 48 h. The cells were stained with Hoechst, and their morphological changes were observed by fluorescence microscope (x400). Arrows show DNA fragmentation in apoptosis in cells. Untreated cells (A); and baicalein-treated cells $20 \mu M(B), 50 \mu M(C)$, and $100 \mu M(D)$.

to conventional therapeutics including surgery, radiation therapy and chemotherapy, which necessitates more intensive treatments that could cause various side effects $(4,13,14)$.

Accordingly, many researchers resort to natural products to avoid side effects. Among multitudinous herbs, flavonoids are becoming more accepted as chemotherapeutic and dietary chemoprevention agents $(11,15,16)$. Furthermore, these natural products have many advantages such as better accessibility and affordability, as well as lower toxicity in comparison with traditional chemotherapy agents. Baicalein is a type of flavonoid and has already been used as an anticancer agent in various cancers such as bladder cancer, breast cancer, cervical cancer, colorectal cancer, gastric cancer, hepatocellular carcinoma, melanoma, skin cancer, osteosarcoma, ovarian cancer, pancreatic cancer, prostate cancer, and lung cancer $(11,12)$. In addition, several preclinical studies have provided evidence that baicalein has the potential to be developed as antitumor drug against cancer by targeting multiple molecular mechanisms and signaling pathways. In our study, the anticancer effects of baicalein in FRO cells were evaluated. Recent findings suggest that one of the common characteristics of spreadable ATCs is the expression of CD133 surface marker es (13). In our study, FRO cells fall in the category of human ATC, and $7 \%$ of FRO cells are CD133-positive.

Induction of cancer cell apoptosis is one of main chemotherapeutic approaches. Apoptosis is active programmed cell death, and this is different from necrosis which represents passive cell death. Accumulating evidence suggests that cancer cell response to chemotherapy is not confined to apoptosis but also to other modes of death (17). Therefore, it is important that baicalein leads ATC cells to die spontaneously via apoptosis because apoptosis is a selfdestructive process of the cell which does not adversely affect 

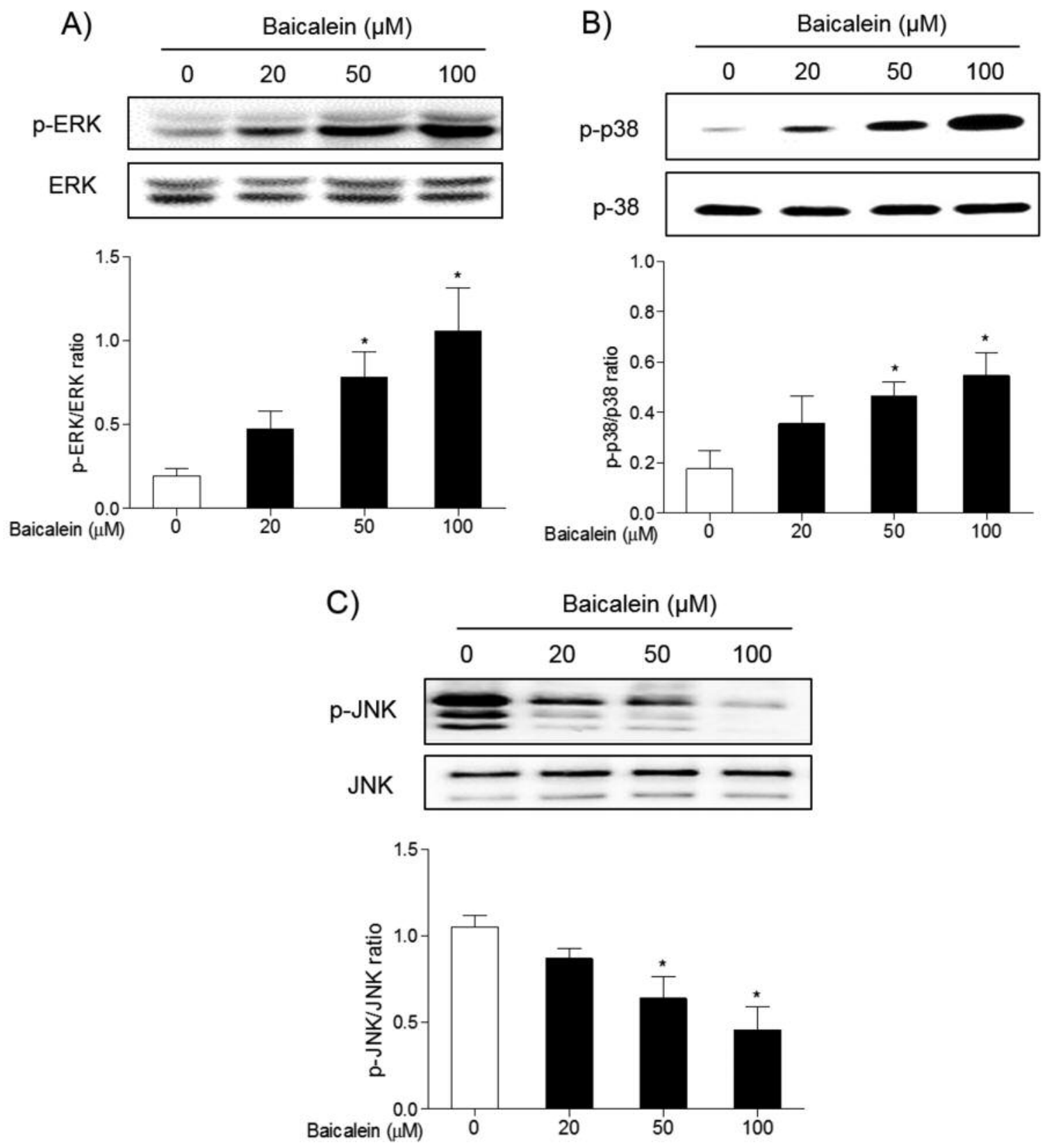

Figure 5. Effects of baicalein on the phosphorylation of ERK, p38, and JNK MAPKs in FRO cells. Cells were treated with 20, 50, and 100 $\mu M$ baicalein for $48 \mathrm{~h}$, and the phosphorylation of ERK (A), p38 (B), and JNK $(C)$ was determined by western blotting. Data are presented as mean $\pm S . E . M$ of the ratio of the intensity of bands of the above proteins vs. the intensity of the bands of their total forms of three independent experiments. ${ }^{*} p<0.05 v s$. normal cells.

other cells or biological processes (18). In general, apoptosis occurs in three ways: granzyme, extrinsic and intrinsic pathway and they all converge to caspase- 3 and the formation of apoptosome (18). The granzyme pathway involves T-cell cytotoxicity. The extrinsic pathway is caspase-dependent and involves the activation of death receptor pathway. The intrinsic pathway is initiated by mitochondria apoptotic pathway. As the stimuli activates the intrinsic pathway, mitochondrial permeability transition pores open and proapoptotic proteins such as PARP and cytochrome $c$ are released and activate multiple caspases. Meanwhile, the Bcl2 family, which regulates the opening of mitochondrial permeability transition pores turns to pro-apoptotic and antiapoptotic depending on the activation ratio of the proapoptotic family such as Bax and the anti-apoptotic family like Bcl-2 $(18,19)$. In our study, 50 and $100 \mu \mathrm{M}$ of baicalein 

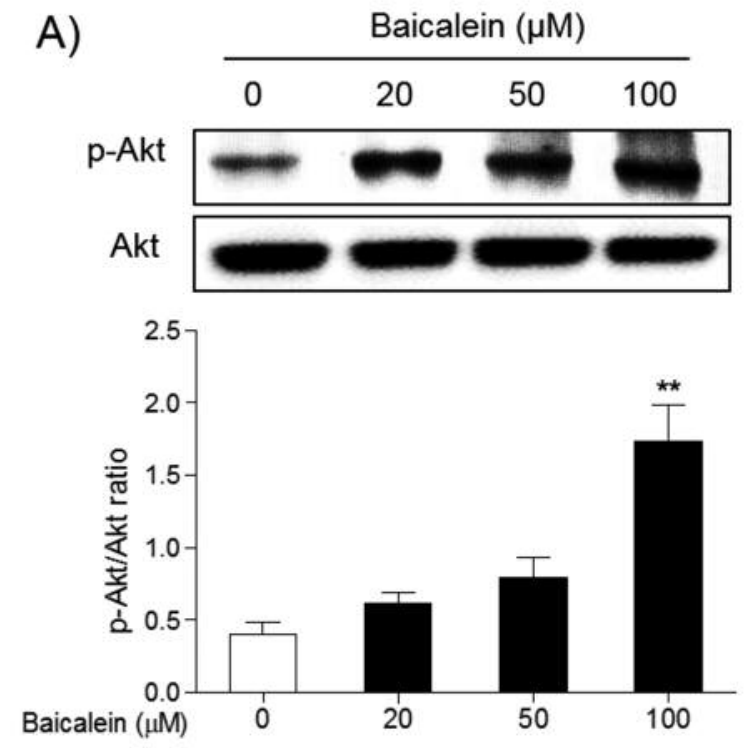

B)
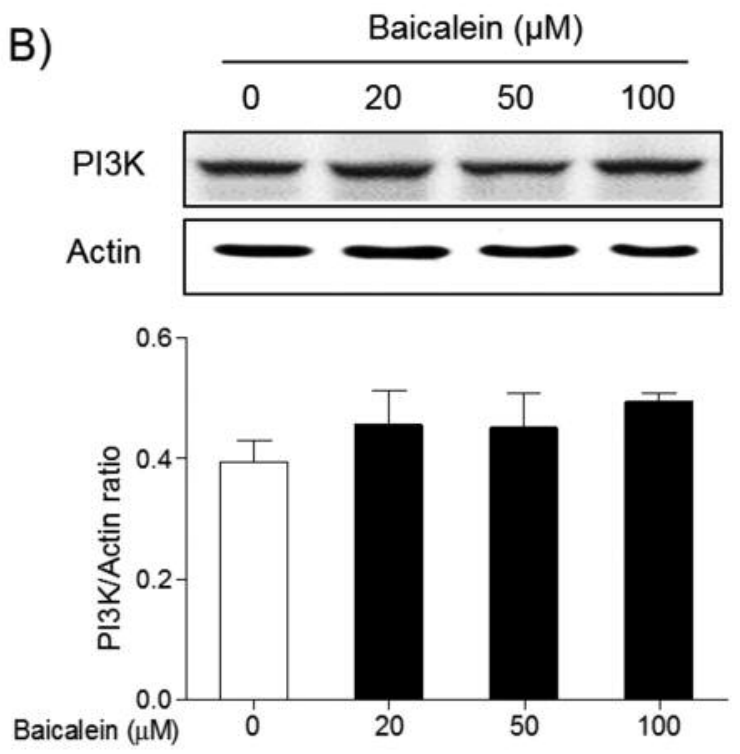

Figure 6. Effects of baicalein on the phosphorylation of Akt and expression of PI3K in FRO cells. Cells were treated with 20, 50, and 100 $\mu M$ baicalein for $48 \mathrm{~h}$, and the phosphorylation of Akt (A) and the expression of PI3K (B) was determined by western blotting. Data are presented as mean \pm S.E.M of the ratio of the intensity of bands of the above proteins vs. the intensity of the bands of total Akt or actin of three independent experiments with total form of Akt or Actin. ${ }^{* *} p<0.01$ vs. normal cells.

significantly decreased the proliferation of FRO cells and induced cell apoptosis. Treatment of FRO cells with baicalein increased the expression of pro-apoptotic proteins, Bax, cytochrome $c$, PARP, and cleaved caspase-3, while decreased the expression of anti-apoptotic protein, Bcl-2 expression. These results indicate that baicalein can induce mitochondrial-mediated apoptosis of ATC through regulation of the expression of pro-apoptotic and anti-apoptotic proteins.

Cox-2 is known to be responsible for invasion and metastasis of cancer cells. The control of the Cox-2/PGE2 pathway suppresses angiogenesis and tumor growth by blocking the VEGF pathway (20). Therefore, Cox-2 inhibition potentiates antiangiogenic cancer therapy and prevents metastasis in preclinical models. In our study, baicalein reduced Cox- 2 expression in FRO cells. This result suggests that baicalein has the potential to prevent invasion and metastasis through inhibition of VEGF-mediated angiogenesis.

The effects of baicalein on the phosphorylation of three MAPK proteins, ERK, JNK, and p38 were also investigated. MAPKs have been associated with regulation of cell fate through apoptosis (21). Recent studies showed that ERK and p38 MAPK activation is necessary for cancer cell death initiated by a variety of anticancer agent $(22,23)$. Meanwhile, JNK is also a regulator of many cellular events including apoptosis. The function of JNK in apoptosis is complex, as has been suggested to have proapoptotic, antiapoptotic, or no role in the process. Some studies show that JNK activation leads to suppression of apoptosis by regulating the $\mathrm{Bcl}-2$ family proteins, $\mathrm{Bcl}-2$, $\mathrm{Bcl}-\mathrm{xL}, \mathrm{Bim}$, and $\mathrm{BAD}$ in response to a variety of extracellular stimuli (24). It is possible that JNK suppresses apoptosis induced by other death insults as well. It is most likely that JNK activation modulates the apoptotic process in a cell type and stimulus dependent manner. However, activation of ERK and p38 MAPK, but not JNK, was found to be involved in baicalein-induced apoptosis of FRO cells, and JNK activation was inhibited by baicalein treatment. This suggests that baicalein-induced apoptosis in ATC involved the phosphorylation of ERK/p38 MAPK.

The MAPK pathway is also closely linked with the PI3K/Akt pathway. The PI3K/Akt pathway has been commonly recognized as an intracellular signaling pathway involved in both normal cell physiology and cancer. In carcinogenesis, the activation of $\mathrm{PI} 3 \mathrm{~K} / \mathrm{Akt} / \mathrm{mTOR}$ pathway induces a protumorigenic phenotype through effects on cell proliferation, cell-cycle progression, inhibition of apoptosis, and angiogenesis (25). Therefore, pharmacological agents are developed and are currently available to specifically target and inhibit PI3K/Akt or mTOR, alone or in combination, and to more effectively treat therapy resistant thyroid cancer (25). Some studies have shown that baicalein inhibits the migration and invasion of cancer cells and induces apoptosis through inhibition of the PI3K/Akt signal pathway $(26,27)$. However, in our study, baicalein inhibited the phosphorylation of Akt, but did not change PI3K. Further studies are required to identify the effects of baicalein on $\mathrm{PI} 3 \mathrm{~K} / \mathrm{Akt} / \mathrm{mTOR}$ signaling pathways in order to understand the anticancer effects of baicalein in ATC.

Our results suggest that baicalein induces apoptosis of FRO cells by increasing the expression of apoptotic proteins, 
Bax, PARP, cytochrome $c$, and caspase-3, inducing the expression of the anti-apoptotic protein, $\mathrm{Bcl}-2$ and activating ERK, and p38 MAPK and Akt signaling pathways.

\section{Conflicts of Interest}

The Authors declare no conflicts of interest regarding the publication of this paper.

\section{Acknowledgements}

This work has been financially supported by Handok Inc.

\section{References}

1 Cabanillas ME, Zafereo M, Gunn GB and Ferrarotto R: Anaplastic thyroid carcinoma: Treatment in the age of molecular targeted therapy. J Oncol Pract 12: 511-518, 2016.

2 Aldinger KA, Samaan NA, Ibanez M and Hill CS Jr.: Anaplastic carcinoma of the thyroid: a review of 84 cases of spindle and giant cell carcinoma of the thyroid. Cancer 41: 2267-2275, 1978.

3 DeSantis CE, Lin CC, Mariotto AB, Siegel RL, Stein KD, Kramer JL, Alteri R, Robbins AS and Jemal A: Cancer treatment and survivorship statistics, 2014. CA Cancer J Clin 64: 252-271, 2014.

4 Lim SM, Shin SJ, Chung WY, Park CS, Nam KH, Kang SW, Keum KC, Kim JH, Cho JY, Hong YK and Cho BC: Treatment outcome of patients with anaplastic thyroid cancer: a single center experience. Yonsei Med J 53: 352-357, 2012.

5 Matuszczyk A, Petersenn S, Voigt W, Kegel T, Dralle H, Schmoll HJ, Bockisch A and Mann K: Chemotherapy with paclitaxel and gemcitabine in progressive medullary and thyroid carcinoma of the follicular epithelium. Horm Metab Res 42: 61-64, 2010.

6 Attar R, Tabassum S, Fayyaz S, Ahmad MS, Nogueira DR, Yaylim I, Timirci-Kahraman O, Kucukhuseyin O, Cacina C, Farooqi AA and Ismail M: Natural products are the future of anticancer therapy: Preclinical and clinical advancements of Viscum album phytometabolites. Cell Mol Biol (Noisy-le-grand) 61: 62-68, 2015.

7 Takahashi H, Chen MC, Pham H, Angst E, King JC, Park J, Brovman EY, Ishiquro H, Harris DM, Reber HA, Hines OJ, Gukovskaya AS, Go VL and Eibl G: Baicalein, a component of Scutellaria baicalensis, induces apoptosis by Mcl-1 downregulation in human pancreatic cancer cells. Biochim Biophys Acta 1813: 1465-1474, 2011.

8 Donald G, Hertzer $\mathrm{K}$ and Eibl G: Baicalein--an intriguing therapeutic phytochemical in pancreatic cancer. Curr Drug Targets 13: 1772-1776, 2012.

9 Wang YF, Xu YL, Tang ZH, Li T, Zhang LL, Chen X, Lu JH, Leung CH, Ma DL, Qiang WA, Wang YT and Lu JJ: Baicalein Induces Beclin 1- and Extracellular Signal-Regulated KinaseDependent Autophagy in Ovarian Cancer Cells. Am J Chin Med 45: 123-136, 2017.

10 Mu J, Liu T, Jiang L, Wu X, Cao Y, Li M, Dong Q, Liu Y and $\mathrm{Xu}$ H: The Traditional Chinese Medicine Baicalein Potently Inhibits Gastric Cancer Cells. J Cancer 7: 453-461, 2016.

11 Gao Y, Snyder SA, Smith JN and Chen YC: Anticancer properties of baicalein: a review. Med Chem Res 25: 1515-1523, 2016.

12 Liu H, Dong Y, Gao Y, Du Z, Wang Y, Cheng P, Chen A and Huang H: The Fascinating Effects of Baicalein on Cancer: A Review. Int J Mol Sci 17: pii:E1681, 2016.
13 Friedman C, Lu M, Schultz A, Thomas D and Lin RY: CD133+ anaplastic thyroid cancer cells initiate tumors in immunodeficient mice and are regulated by thyrotropin. PLos One 4: e5395, 2009.

14 Adkins D, Ley J, Trinkaus K, Thorstad W, Lewis J Jr, Wildes T, Siegel BA, Dehdashti F, Gay H, Mehan P and Nussenbaum B: A phase 2 trial of induction nab-paclitaxel and cetuximab given with cisplatin and 5-fluorouracil followed by concurrent cisplatin and radiation for locally advanced squamous cell carcinoma of the head and neck. Cancer 119: 766-773, 2013.

15 Romano B, Pagano E, Montanaro V, Fortunato AL, Milic N and Borrelli F: Novel insights into the pharmacology of flavonoids. Phytother Res 27: 1588-1596, 2013.

16 Yao H, Xu W, Shi X and Zhang Z: Dietary flavonoids as cancer prevention agents. J. Environ. Sci. Health C Environ. Carcinog Ecotoxicol Rev 29: 1-31, 2011.

17 Brown JM and Attardi LD: The role of apoptosis in cancer development and treatment response. Nat Rev Cancer 5: 231237, 2005.

18 Zhou QM, Wang S, Zhang H, Lu YY, Wang XF, Motoo Y and Su SB: The combination of baicalin and baicalein enhances apoptosis via the ERK/p38 MAPK pathway in human breast cancer cells. Acta Pharmacol Sin 30: 1648-1658, 2009.

19 Elmore S: Apoptosis: a review of programmed cell death. Toxicol Pathol 35: 495-516, 2007.

$20 \mathrm{Xu}$ L, Stevens J, Hilton MB, Seaman S, Conrads TP, Veenstra TD, Loqsdon D, Morris H, Swing DA, Patel NL, Kalen J, Haines DC, Zudaire E and St Croix B: COX-2 inhibition potentiates antiangiogenic cancer therapy and prevents metastasis in preclinical models. Sci Transl Med 6: 242ra84, 2014.

21 Wada T and Penninger JM: Mitogen-activated protein kinases in apoptosis regulation. Oncogene 23: 2838-2849, 2004.

$22 \mathrm{Yu}$ JS and Kim AK: Wogonin induces apoptosis by activation of ERK and p38 MAPKs signaling pathways and generation of reactive oxygen species in human breast cancer cells. Mol Cells 31: 227-335, 2011.

23 Min S, More SV, Park JY, Jeon SB, Park SY, Park EJ, Yoon SH and Choi DK: EOP, a newly synthesized ethyl pyruvate derivative, attenuates the production of inflammatory mediators via p38, ERK and NF- $x \mathrm{~B}$ pathways in lipopolysaccharide-activated BV-2 microglial cells. Molecules 19: 19361-19375, 2014.

24 Yu C, Minemoto Y, Zhang J, Liu J, Tang F, Bui TN, Xiang J and Lin A: JNK suppresses apoptosis via phosphorylation of the proapoptotic Bcl-2 family protein BAD. Molecular Cell 13: 329340, 2004.

25 Petrulea MS, Plantinga TS, Smit JW, Georgescu CE and NeteaMaier RT: PI3K/Akt/mTOR: A promising therapeutic target for nonmedullary thyroid carcinoma. Cancer Treat Rev 41: 707-713, 2015.

26 Zhang HB, Lu P, Guo QY, Zhang ZH and Meng XY: Baicalein induces apoptosis in esophageal squamous cell carcinoma cells through modulation of the PI3K/Akt pathway. Oncol Lett 5: 722728, 2013.

27 Choi EO, Cho EJ, Jeong JW, Park C, Hong SH, Hwang HJ, Moon SK, Son CG, Kim WJ and Choi YH: Baicalein Inhibits the Migration and Invasion of B16F10 Mouse Melanoma Cells through Inactivation of the PI3K/Akt Signaling Pathway. Biomol Ther (Seoul) 25: 213-221, 2017.

Received October 30, 2018

Revised November 14, 2018

Accepted November 16, 2018 